\title{
Random Forest for Reliable Pre-Classification of Handwritten Characters
}

\author{
L.P. Cordella \\ Dip. di Ing. Elettrica e delle Tecnologie dell'Informazione (DIETI) \\ Università di Napoli Federico II \\ Napoli, Italy \\ cordel@unina.it
}

\author{
C. De Stefano, F. Fontanella, A. Scotto di Freca \\ Dip. di Ing. Elettrica e dell'Informazione (DIEI) \\ Università di Cassino e del Lazio meridionale \\ Cassino (FR), Italy \\ \{destefano, fontanella, a.scotto $\} @$ unicas.it
}

\begin{abstract}
In many real world applications, the cost of a wrong decision may be much higher than the benefit of having a high classification rate. This kind of classification problems represent very challenging tasks, because they require highly reliable systems and may benefit of introducing a reject option. To this purpose, many classification systems have been proposed, among which classifier ensembles represent a successful example. This approach aims at combining classifiers making uncorrelated errors. In this framework, the Random Forest (RF) represents a case of special interest. A RF is made of a suitable ensemble of decision trees and has proved to be very effective in several fields. In this paper, the RF classification reliability is experimentally analyzed with reference to the case of handwriting recognition. The aim is to verify if such reliability can be effectively used to introduce a new reject option, which considers, for each unknown sample, a subset of few classes including with high probability the correct one. The whole system operates as a pre-classification stage and the reject option should allow us to obtain a low error rate without significantly affecting the recognition rate. Experiments, carried out on two real world datasets, have shown the effectiveness of the proposed method.
\end{abstract}

\section{INTRODUCTION}

Many real world applications, such as medical diagnosis, fraud detection, postal sorting, etc. are cost-sensitive classification problems: the loss caused by a wrong prediction may be substantially greater than the advantage due to the correct one. These problems are usually solved by using classifiers that provide an estimate of the probability that the input sample belongs to the class it has been assigned to. This probability may be assumed as a measure of classification reliability. Once this reliability measure is available, a reject option can be implemented by rejecting those samples whose reliability measure is below a certain threshold. Of course, the choice of the reject threshold is a critical problem and many studies have been devoted to it in the literature. In particular, in [1] the problem of achieving the optimal trade-off between error and reject rates is addressed from a probabilistic point of view. The author shows that the error rate decreases monotonically as the rejection rate increases. Based on this relationship, the author proves that, if the conditional probability densities are exactly known, then the optimal error versus rejection trade-off can be found.

Unfortunately, in real applications, these densities are generally unknown. In [2], [3], [4], [5] a number of strategies for defining an optimal rejection rule can be found. However, in cases in which many samples are located in regions of the feature space where two or more classes overlap, any attempt to reject misclassified samples also implies the rejection of a significant number of correctly classified samples.

To deal with this problem, a useful approach could be that of not requiring to obtain a single class as output of the classifier, but to accept to drastically reduce the number of initial classes, by using the classifier as a pre-classification stage providing the set of the $n$ most probable classes. Of course $n$ should be as small as possible. In such a way, the classification complexity is reduced since, in principle, it can be assumed that choosing among few classes, at a second stage of classification, is simpler than choosing among many classes.

This approach has been used in applications like speech and character recognition. In [6], for example, decision trees are used to build a high performance system for Optical Character Recognition (OCR) pre-classification, whereas in [7] a preclassifier is used to improve the performance of a hidden Markov model classifier for cursive words. In [8], the rejection problem is extended to the case in which a subset of classes is provided as response by a classifier and a reject rule which finds the optimal trade-off between error and number of classes is proposed.

This approach has been also used in the framework of cursive word recognition problems. In this context, in fact, a useful approach may be that of identifying and classifying the parts of the ink which possibly correspond to single characters. In case of analytical approaches [9], the detection of each single character within the word is obviously fundamental, since they consider the entries as possible strings of characters and use this information to either validate/reject the class assigned to each character by the underlying OCR, or to select the best string among many possible alternatives. Even if, in principle, these approaches should allow us to read any word, in practice the use of a dictionary strongly increases the obtainable performance. Also in case of holistic approaches [9], where the whole word is the pattern to classify and the number of classes is equal to the entries in the dictionary, there are often disconnected parts of the ink which correspond to isolated characters: the classification of such characters may be very helpful for increasing the probability of correctly recognizing the word, since it may significantly reduce the number of entries to be considered in the dictionary. In both cases, the cost of an error may be very high and may result in misclassifying the whole word. On the contrary, providing for each character a small set of classes, which includes with 
high probability the correct class, do not increase the error rate, but may improve the whole word recognition rate. The only negative effect is the increase of the computational cost of the whole word classification process.

Among the many classification systems available, Multiple Classifier Systems (MCS) have shown to be particularly effective for dealing with complex classification problems. These systems are made of sets of classifiers and combine their responses in the attempt to get better performance than that achieved by each single component. Nowadays, it has been widely recognized that performance improvements strongly depend on the diversity of the classifiers making up the system, so as to obtain uncorrelated prediction errors.

Among MCS, Classifier Ensembles (CE) are made of classifiers based on the same classification scheme, but using different training approaches. Two such approaches have proved to be very successful [10], [11]: (i) training the single classifiers on different data subsets (ii) using different feature subsets for each classifier. Bagging [12] and boosting [13] techniques belong to the first category, whereas the Random subspaces approach [14] falls into the second one. In the bagging approach, different data subsets are obtained by performing a random sampling with replacement on the data set: each classifier is then trained on a different subset. The boosting technique, instead, uses an iterative training procedure: the ensemble classifiers are trained sequentially and the training set for each classifier is more likely to contain those samples that have been misclassified by the previously trained classifier. The Random Subspace method belongs to the second category and produces diversity by training each classifier on a different feature subset, built by randomly selecting a subset of all the available features.

In [15], a new algorithm for building decision tree ensembles named Random Forest (RF) is presented, which combines bagging [12] and Random Subspace methods in order to maximize the diversity among the trees making up the ensemble. In practice, in the RF algorithm, each ensemble tree is built by using both a different training set (because of bagging) and a different feature subset (because of Random Subspace). Once the ensemble has been built, unknown samples are labeled according to the majority vote rule. Since its presentation, the RF approach has attracted the attention of researchers. Many studies have been conducted, both for comparing its performance with those of other ensemble methods and for exploiting its effectiveness in practical applications [16], [17], [18], [19], [20]. In [15] and [10] it was shown that RF outperforms both bagging and boosting. As regards practical applications, RF have proved to be very effective in the field of image analysis. In particular, in [16] RF is used for video object segmentation, while in [17] RF is employed for hyperspectral data classification. In [20] the RF global behavior is analyzed with respect to the parameter settings.

It is worth noticing that, although RF has been widely used for various applications, it has been never used with a reject option in multi-class classification problems.

The aim of this paper is twofold. First, we performed an experimental analysis of Random Forest with the aim of understanding to what an extent the classification reliability measure made available by such algorithm (class probability) can be used to implement an effective reject option.
Second, we used the set of class probabilities given by the RF to develop a pre-classification system whose aim is that of selecting, for each unknown sample, a subset of few classes, which includes with high probability the correct one. Such a subset can be then considered by a second-stage classifier to perform the final classification.

In the framework of a cursive word recognition system for postal applications, we tested the proposed approach to the handwritten character classification problem, which involves fifty-two different classes. As previously discussed, in this case the selection of a small subset of possible classes may be an effective strategy for improving the overall recognition rate. Moreover, the cost of a rejection is much lower than that of an error: in fact, in case of an error, i.e. the correct class is not present in the subset provided for one of the characters in a word, that word will be certainly misclassified. On the contrary, in case of a rejection, using the other information extracted from the word, it is still possible to obtain a correct classification. In the experiments, we have shown how the overall error rate varies as a function of both the reject threshold value and the number of classes in the selected subsets.

The remainder of the paper is organized as follows. In Section II the Random Forest algorithm is detailed; in this section we also explain how output class probabilities are estimated. In Section III the experimental results are shown, while Section IV reports the concluding remarks and the aspects on which the future work will focus.

\section{RANDOM FOREST}

The term "Random Forest" refers to a general methodology for building an ensemble of $L$ tree-based classifiers $\left.\left\{T_{i}\left(\mathbf{x}, \boldsymbol{\Theta}_{\mathbf{i}}\right)\right)\right\}$, where $\mathbf{x}$ is an input feature vector and $\boldsymbol{\Theta}_{\mathbf{i}}$ is a random vector that governs the growth of the $i$ th tree. The random vector $\Theta_{i}$ is generated independently of the preceding $\Theta_{1} \ldots \Theta_{i-1}$, but with the same distribution. In bagging, for instance, the random vector is $\boldsymbol{\Theta}_{\mathbf{i}}=\left\{\theta_{1}, \theta_{2} \ldots, \theta_{N}\right\}$ where $N$ is the training set size and $\theta_{j}$ is an integer value randomly drawn (with uniform distribution) from the set $\{1,2, \ldots, N\}$. In the random subspace method, instead, $\Theta_{\mathbf{i}}$ consists of a $K$ integers $(K \ll M)$ randomly drawn from a uniform distribution in the interval $[1, M]$, where $M$ is the number of available features. The Random Forest approach does not refer to a single algorithm, but rather to a family of methods. In this study, we have used the original algorithm proposed by Breiman in [15], usually referred in the literature as Forest$R I$. For its effectiveness, this algorithm was used as reference method in most of the papers dealing with RF. Given a dataset set that contains $N$ feature vectors, each consisting of $M$ features, the forest-RI algorithm consists of the following steps:

1) Draw, from the dataset, $N$ samples at random with replacement. The resulting set will be the training set of the tree.

2) Set a number $K \ll M$

3) At each node, randomly draw $K$ features from the set of available features.

4) Among the values of each of the $K$ features drawn, choose the best binary split according to the Gini index [21]. 


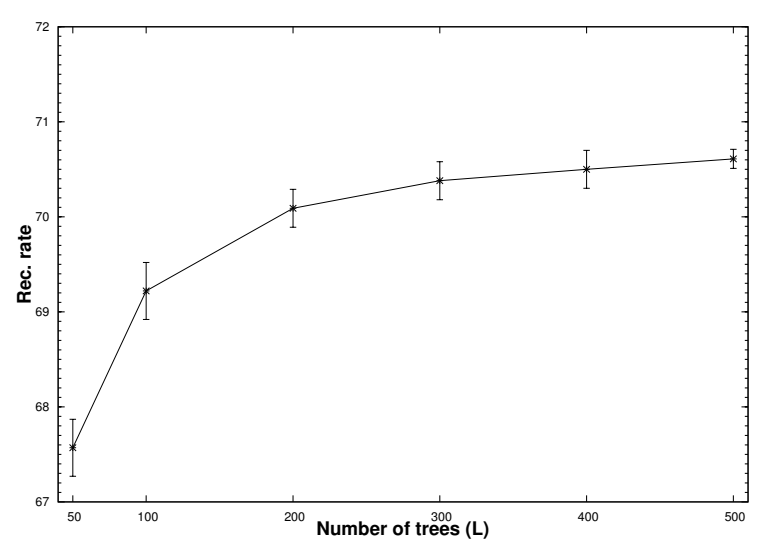

(a)

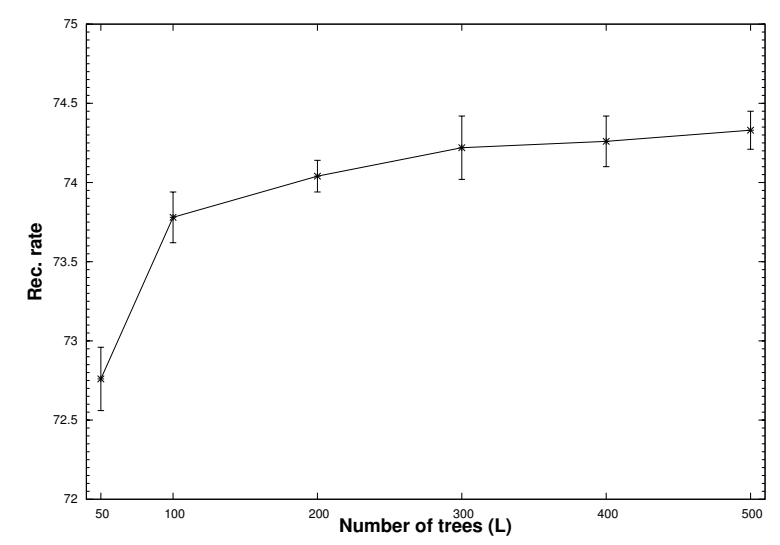

(b)

Fig. 1. Accuracy on the test set as a function of the number of trees in the ensemble, for the NIST database (a) and the PD database (b).

5) Grow the tree to its maximum size according to the stopping criterion chosen ${ }^{1}$.

6) Let the tree unpruned.

Once the forest has been built, an unknown sample is labeled according to the Majority Vote rule: i.e., it is labeled with the most popular class among those provided by the ensemble trees. It is worth noting that in [12] it is proved that RF does not over-fit as more trees are added, rather its generalization error tends to a limiting value.

\section{A. Class Probability Estimation in Random Forest}

In the RF approach, given a sample $\mathbf{x}$ to be classified, the conditional probabilities for each class are computed by averaging the conditional probabilities given by the trees making up the ensemble. These conditional probabilities are computed as follows. Given a generic decision tree $T$, and an input sample $\mathrm{x}$ to be recognized, let us denote by $v(x)$

\footnotetext{
${ }^{1}$ Node splitting usually is stopped when one of the following conditions occur: (i) The number of samples in the node to be split is below a given threshold; (ii) all the samples in the node belong to the same class
}

the leaf node where $x$ falls when it is classified by $T$. The probability $P(c \mid \mathbf{x}, T)$ that the sample $\mathbf{x}$ belongs to the class $c(c \in\{1,2, \ldots, C\})$, is estimated by the following equation:

$$
P(c \mid \mathbf{x}, T)=\frac{n(c)}{n}
$$

where $n(c)$ is the number of training samples belonging to the class $c$ falling into $v(x)$, while $n$ is the total number of training samples falling into $v(x)$. Both numbers are available at the end of the learning procedure.

Given a RF consisting of $L$ trees and an unknown sample $\mathbf{x}$ to be classified, the probability estimate $P(c \mid \mathbf{x})$ that $\mathbf{x}$ belongs to the class $c$ is computed as follows:

$$
P(c \mid \mathbf{x})=\frac{1}{L} \sum_{i=1}^{L} P\left(c \mid \mathbf{x}, T_{i}\right)
$$

where $P\left(c \mid \mathbf{x}, T_{i}\right)$ is the conditional probability provided by the $i$ th tree and is computed according to eq. (1). As a consequence, for the sample $\mathbf{x}$ to be classified, the RF algorithm gives as output the vector:

$$
\mathbf{p}=\{P(1 \mid \mathbf{x}), P(2 \mid \mathbf{x}), \ldots, P(C \mid \mathbf{x})\}
$$

\section{B. Reject rules}

A reject rule may be simply implemented by taking into account the class probability estimation provided by the random forest. In practice, for an unknown sample $\mathbf{x}$, denoting as $c$ the label of the class corresponding to the higher value $P(c \mid \mathbf{x})$ in the output vector $\mathbf{p}$ (see eq. 3), the response of the RF is accepted if $P(c \mid \mathbf{x}) \geq T_{r}$, otherwise it is rejected. $T_{r}$ is called reject threshold and assumes values in the range $[0.0,1.0]$. When the RF algorithm is used as a pre-classifier system, i.e. providing as output a subset of the $m$ most likely classes, rather than the most likely one, a reject rule may be implemented as follows: Let $T_{r} \in[0.0,1.0]$ the reject threshold and $\mathbf{p}=\{P(1 \mid \mathbf{x}), P(2 \mid \mathbf{x}), \ldots, P(C \mid \mathbf{x})\}$ the class probability vector provided as output by the RF for an input sample $\mathbf{x}$, the $m$ classes $(m>1)$ are obtained as follows:

1) sort $\mathbf{p}$ in decreasing order.

2) Let $\mathbf{p}^{\prime}=\{P(\pi(1) \mid \mathbf{x}), P(\pi(2) \mid \mathbf{x}), \ldots, P(\pi(C) \mid \mathbf{x})\}$ the sorted vector, $(P(\pi(i)) \geq P(\pi(i+1)), 1 \leq i \leq C-1)$.

3) Calculate the sum $p_{s}=\sum_{i=1}^{m} P(\pi(i))$

4) If $p_{s} \geq T_{r}$ then gives as output the subset of classes: $\{\pi(1), \pi(2)$, $\ldots, \pi(m)\}$

else reject the sample

Summarizing, the rule rejects all the samples for which the sum of the probabilities of the $m$ most likely classes is below the reject threshold.

\section{EXPERIMENTAL RESULTS}

In order to assess the performance of Random Forest in handwritten character Recognition, with special reference to applications where the cost of an error is high, two real word datasets were taken into account: the well known NIST-SD19 


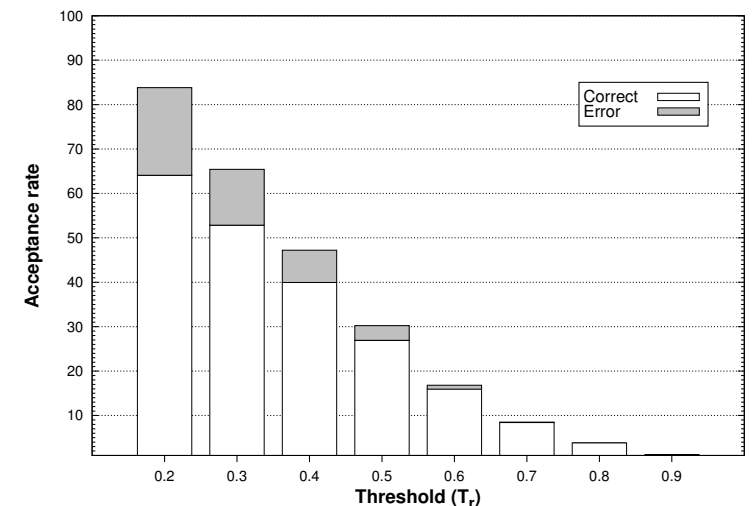

(a)

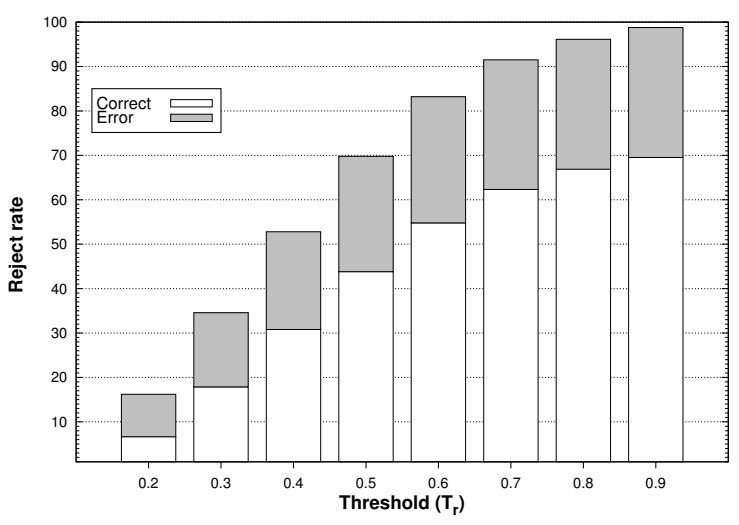

(b)

Fig. 2. Acceptance rate (a) and reject rate (b) as a function of the reject threshold for the NIST test set. In each bar the gray part indicates the percentage of rejected samples that would be misclassified without applying the reject option, while the white part indicates the same information for the correctly classified samples.

(NIST in the following) and a dataset of characters segmented from postal addresses (PD in the following).

The NIST dataset [22] contains binary images representing alphanumeric characters. In particular, we have considered handwritten uppercase and lowercase letters (52 classes). The handwriting sample form $h s f 4$, containing 23941 characters (11941 uppercase and 12000 lowercase), has been used as training set, while the form $h s f 7$, containing 23670 characters (12092 uppercase and 11578 lowercase) has been used as test set. In each form, characters are isolated, labeled and stored in $128 \times 128$ pixel images.

The PD dataset, made of variable size images, has been divided into a training set, made of 5293 lowercase and 2739 uppercase letters and a test set made of 5285 lowercase and 2747 uppercase letters. In both datasets, samples are represented by using the features proposed in [23]. Each character image is divided into six parts and is described by a feature vector containing measures associated to the different parts. For each part, 22 features are computed, for a total of 132 features: 13 of these features represent concavity measures, while the

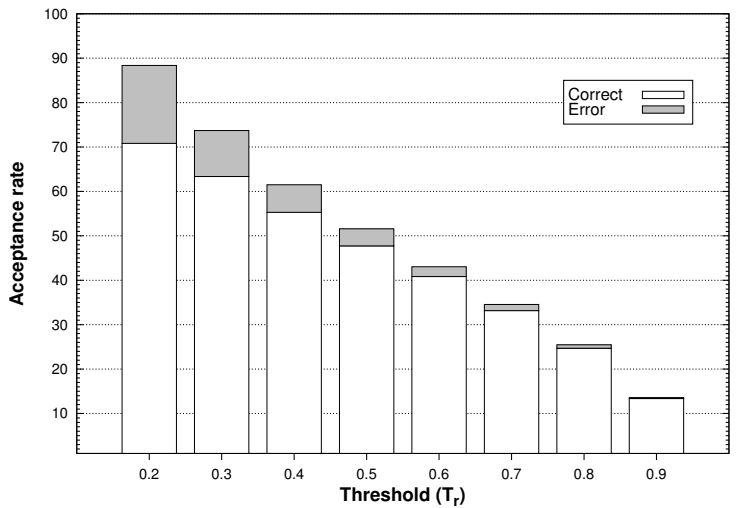

(a)

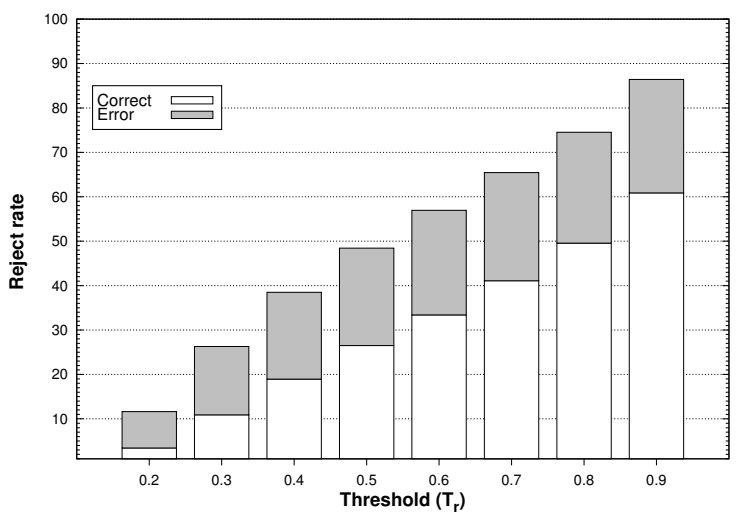

(b)

Fig. 3. Acceptance rate (a) and reject rate (b) as a function of the reject threshold for the PD test set. In each bar the gray part indicates the percentage of rejected samples that would be misclassified without applying the reject option, while the white part indicates the same information for the correctly classified samples.

remaining ones are related to other contour information.

To deal with the randomness of the RF algorithm, due to bagging and random subspace procedures, 20 runs have been performed for each experiment carried out. All the results reported in the following were obtained by averaging the values over the 20 runs. The parameter $K$ (number of randomly selected features at each node split), was set to $\left\lceil\log _{2}(N)\right\rceil$, where $N$ is the training set size. This value is the same chosen by Breiman in the experiments reported in [15] and has often been used in the literature as default value.

In Fig. 1 the accuracy achieved on the test set as function of the number of trees in the ensemble (parameter $L$ ) is shown. The plots also include the related standard deviations. Six increasing values in the range $[50,500]$ were tested. The aim of this experiment is to investigate the effects of the parameter $L$ on the RF accuracy. From the plots it can be seen that, as expected, the higher $L$, the higher the test set accuracy, though the accuracy increment decreases as the $L$ value increases. In order to assess whether these performance 


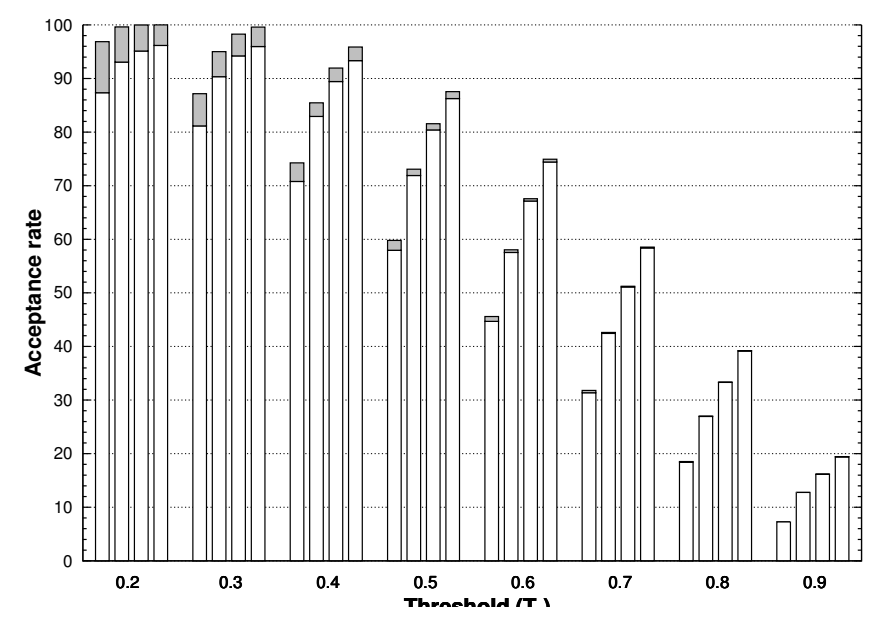

(a)

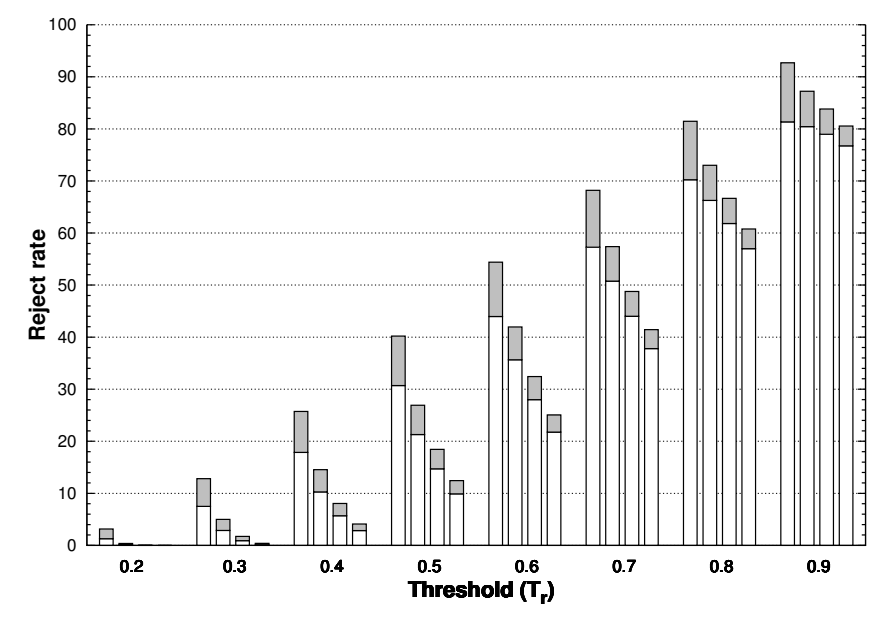

(b)

Fig. 4. Acceptance rate (a) and reject rate (b) as a function of the reject threshold for the NIST test set, for $m \in[2,3,4,5]$. For each threshold, bars are sorted from left to right by increasing values of $m$. Gray and white respectively indicate correctly classified and misclassified samples.

differences were statistically significant, a $t$-test $(\alpha=0.05)$ was performed. For both datasets, the tests confirmed that the performance differences are statistically significants only for the values of $L \leq 300$. Therefore, with the purpose of keeping the learned RF model as simple as possible, but maximizing the performance, in the experiments described in the following the value for $L$ was set to 300 . Under these conditions, the error rate obtained for the NIST database was $29.6 \%$ and that for the PD database was $25.8 \%$.

A further experiment investigated how the introduction of the reject option affects both the recognition rate and the error rate. Fig. 2 and Fig. 3 show the trend of both the acceptance rate and the reject rate as a function of the reject threshold. It is evident that as the reject threshold value increases, the number of rejected samples that would be correctly classified without

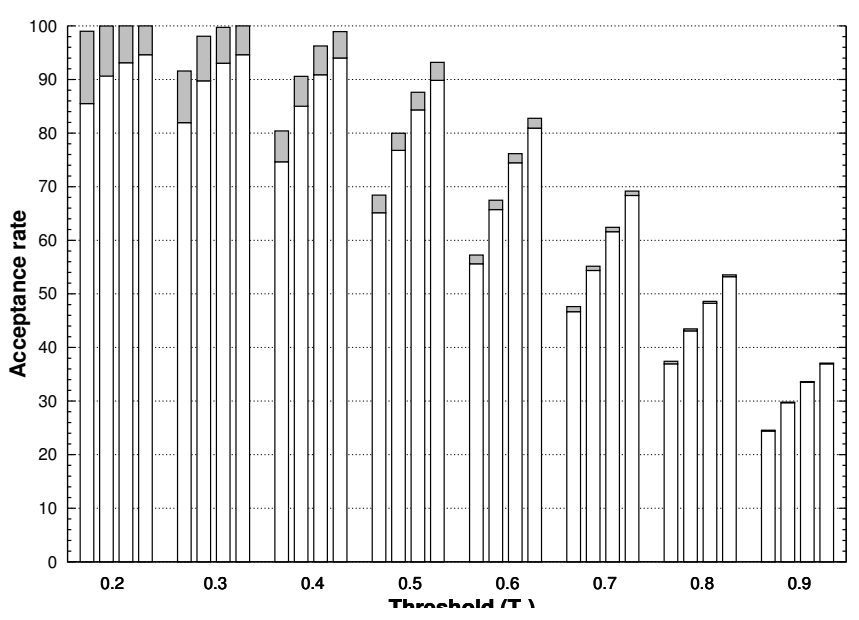

(a)

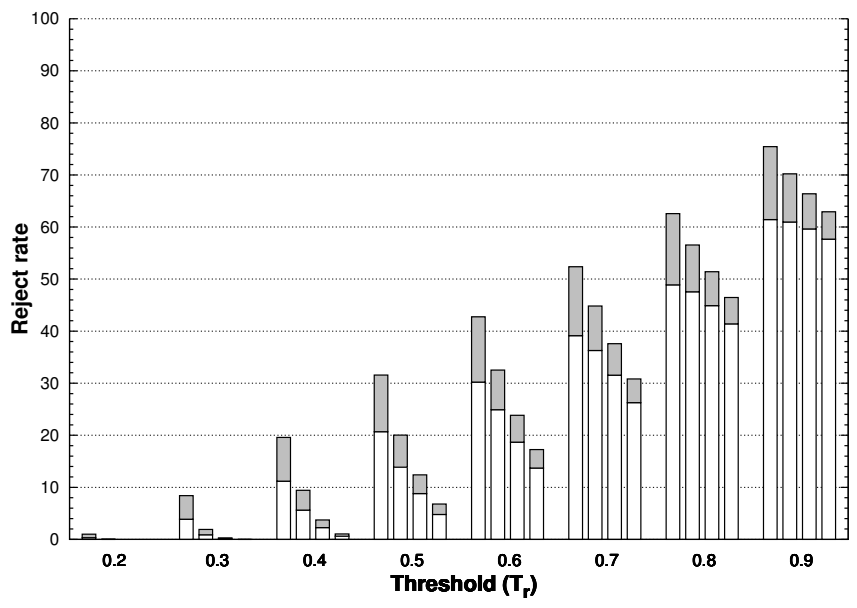

(b)

Fig. 5. Acceptance rate (a) and reject rate (b) as a function of the reject threshold for the PD test set, for $m \in[2,3,4,5]$. For each threshold, bars are sorted from left to right by increasing values of $m$. Gray and white respectively indicate correctly classified and misclassified samples.

the rejection option significantly increases, while the number of samples misclassified without the rejection option quickly tends to a maximum and remains constant. From the figures, it appears that the introduction of a reject option does not allow us to obtain satisfactory results. In fact, for the NIST database, a reduction of the error rate below $10 \%$ requires the rejection of more than $50 \%$ of samples, with a correct recognition rate equal to about $40 \%$. Moreover, for $T_{r}$ values greater than 0.3 , a small reduction of the error rate implies a large reduction of the recognition rate. Similar considerations hold for the PD database.

In order to overcome the problems due to the unfeasible recognition rates obtained by applying the reject option, we analyzed the performance of the RF system when used as a pre-classifier, i.e providing as output not just one class, but a 
small subset of $m$ possible classes ( $m \ll C$ ). Note that $m$ is a value that can be a priori set by the user. In the experiments, values of $m$ ranging from 2 to 5 were considered.

Fig. 4 and Fig. 5 show the plots of the acceptance rate and the reject rate as a function of the reject threshold for the NIST and the PD databases, respectively. For each threshold value, there are four bars representing the results obtained by using values of $m$ ranging from 2 to 5 , respectively.

From the plots it can be observed that, for $T_{r} \geq 0.5$, the error rate is low and exhibits slight variations, while the recognition rate rapidly decreases. As a consequence, for $T_{r} \geq 0.5$, the majority of the rejections are those samples that would have been correctly classified without applying the reject option. The pre-classification system operates in these conditions when there are severe requirements on the error rate. Consider, for instance, the case reported in Fig. 5: in order to assure an error rate less than $0.5 \%$, the value 0.8 must be used for $T_{r}$, which implies a recognition rate of about $55 \%$ with $m=5$. From the plots it is also evident that, for $T_{r} \leq 0.5$, the introduction of the reject option allowed us to obtain very interesting results, specially for $m$ equal to 4 or 5 : in these cases, in fact, we obtained a recognition rate equal to about $95 \%$, with a reduction lower than $4 \%$, maintaining the error rate below $5 \%$ (see Fig. 4 with $T_{r}$ equal to 0.3 and 0.4 , and Fig. 5 with $T_{r}$ equal to 0.4 and 0.5 ).

\section{CONCLUSIONS}

In this paper, an experimental study of Random Forest reliability in handwritten character recognition has been proposed. Two problems have been investigated: i) the relationship between error rate and rejection rate; ii) the Random Forest performance in pre-classification tasks. The study has been performed by using a specifically devised rejection option that takes into account the class probability estimations provided by the Random Forest. Experiments were carried out on two real world datasets, containing handwritten uppercase and lowercase letters, totaling 52 classes. The experimental results, obtained using two different quite large databases, can be summarized as follows: (i) when Random Forest is used as a classifier (i.e. it provides in output just one class) the introduction of a reject option produces low error rate only when a reject rate greater than $50 \%$ is accepted; (ii) when Random Forest is used as pre-classification stage, the introduction of the reject option allows us to significantly lower the error rate, without being forced to accept high rejection rates. It has to be remarked that, when few classes are suggested by the pre-classifier, the following classification stage may be very simple to design, since often a few distinctive features may be sufficient for discriminating among the classes. Moreover, in cursive handwriting recognition problems, which often require the use of a dictionary, the selection of a small subset of possible classes, which includes with high probability the correct one, may be an effective strategy for improving the overall recognition rate. In this case, in fact, an error on a single character makes it impossible to select the correct entry from the dictionary.

Future work will include: (i) experiments on larger datasets; (ii) the use of different reject rule, e.g., the Class-Selective Reject Rule; (iii) the implementation of a second-stage classifier.

\section{REFERENCES}

[1] C. Chow, "On optimum recognition error and reject trade off," IEEE Trans. Inf. Theor., vol. 16, no. 1, pp. 41-46, 2006.

[2] L. Cordella, C. De Stefano, F. Tortorella, and M. Vento, "A method for improving classification reliability of multilayer perceptrons," Neural Networks, IEEE Transactions on, vol. 6, no. 5, pp. 1140 -1147, 1995.

[3] C. De Stefano, C. Sansone, and M. Vento, "To reject or not to reject: that is the question-an answer in case of neural classifiers," Systems, Man, and Cyber. Part C, IEEE Transactions on, vol. 30, no. 1, pp. 84-94, 2000.

[4] T. Landgrebe, D. M. J. Tax, P. Paclík, and R. P. W. Duin, "The interaction between classification and reject performance for distancebased reject-option classifiers." Pattern Recognition Letters, vol. 27, no. 8, pp. 908-917, 2006.

[5] B. Hanczar and E. R. Dougherty, "Classification with reject option in gene expression data." Bioinformatics, vol. 24, no. 17, pp. 1889-1895, 2008.

[6] H. Baird and C. L. Mallows, "Bounded-error preclassification trees," in Shape, Structure and Pattern Recognition, D. Dori and A. Bruckstein, Eds. World Scientific, 1995, pp. 100-110.

[7] W. Cho, S.-W. Lee, and J. H. Kim, "Modeling and recognition of cursive words with hidden markov models," Pattern Recognition, vol. 28, no. 12, pp. 1941-1953, 1995.

[8] T. Ha, "The optimum class-selective rejection rule," Pattern Analysis and Machine Intelligence, IEEE Transactions on, vol. 19, no. 6, pp. $608-615,1997$.

[9] R. Plamondon and S. Srihari, " On-line and off-line handwriting recognition: A comprehensive survey ," IEEE Trans. Pattern Anal. Mach. Intell., vol. 22, no. 2, pp. 63-84, 2000.

[10] L. I. Kuncheva, Combining Pattern Classifiers: Methods and Algorithms. Wiley-Interscience, 2004.

[11] T. G. Dietterich, "An Experimental Comparison of Three Methods for Constructing Ensembles of Decision Trees: Bagging, Boosting, and Randomization," Machine Learning, vol. 40, pp. 139-157, 2000.

[12] L. Breiman, "Bagging Predictors," Machine Learning, vol. 24, no. 2, pp. 123-140, 1996.

[13] Y. Freund and R. Shapire, "Experiments with a new boosting algorithm," in Proc. of the 13th Int. Conf. on Machine Learning, 1996, pp. 148-156.

[14] T. K. Ho, "The Random Subspace Method for Constructing Decision Forests," IEEE Trans. Pattern Anal. Mach. Intell., vol. 20, no. 8, pp. 832-844, 1998.

[15] L. Breiman, "Random Forests," Machine Learning, vol. 45, no. 1, pp. 5-32, 2001

[16] H.-T. Chen, T.-L. Liu, and C.-S. Fuh, "Segmenting highly articulated video objects with weak-prior random forests," in Proc. of ECCV'06. Berlin, Heidelberg: Springer-Verlag, 2006, pp. 373-385.

[17] J. Ham, Y. Chen, M. M. Crawford, and J. Ghosh, "Investigation of the random forest framework for classification of hyperspectral data," IEEE Trans. Geoscience and Remote Sensing, vol. 43, no. 3, pp. 492-501, 2005.

[18] M. Crawford, J. Ham, Y. Chen, and J. Ghosh, "Random forests of binary hierarchical classifiers for analysis of hyperspectral data," in Advances in Techniques for Analysis of Remotely Sensed Data, 2003 IEEE Workshop on, 2003, pp. 337-345.

[19] S. R. Joelsson, J. A. Benediktsson, and J. R. Sveinsson, "Feature Selection for Morphological Feature Extraction using Random Forests," in Proc. of NORSIG 2006, 2006, pp. 10-13.

[20] S. Bernard, L. Heutte, and S. Adam, "Using Random Forests for Handwritten Digit Recognition," in Proc. of ICDAR 2007, vol. 2, 2007, pp. 1043-1047.

[21] C. Gini, "Measurement of inequality of incomes," The Economic Journal, vol. 31, no. 121, pp. 124-126, 1921.

[22] J. Grother, "NIST special database 19: Hand printed forms and characters database," Tech. Rep., 1995.

[23] L. Oliveira, R. Sabourin, F. Bortolozzi, and C. Suen, "Automatic recognition of handwritten numerical strings: A recognition and verification strategy," IEEE Trans. Pattern Analysis and Machine Intelligence, vol. 24, no. 11, pp. 1438-1454, 2002. 